\title{
Escala de Autoestima de Rosenberg (EAR): validade fatorial e consistência interna
}

\author{
Juliana Burges Sbicigo' - Universidade Federal do Rio Grande do Sul, Porto Alegre, Brasil \\ Denise Ruschel Bandeira - Universidade Federal do Rio Grande do Sul, Porto Alegre, Brasil \\ Débora Dalbosco Dell'Aglio - Universidade Federal do Rio Grande do Sul, Porto Alegre, Brasil
}

\begin{abstract}
Resumo
O objetivo deste estudo foi investigar as propriedades psicométricas da Escala de Autoestima de Rosenberg (EAR) para adolescentes. Participaram 4.757 adolescentes, com idades entre 14 e 18 anos $(M=15,77 ; D P=1,22)$, de nove cidades brasileiras. Os participantes responderam a uma versão da EAR adaptada para o Brasil. A análise fatorial exploratória apontou uma estrutura bidimensional, com $51.4 \%$ da variância explicada, que foi sustentada pela análise fatorial confirmatória. As análises de consistência interna realizadas por meio do coeficiente alfa de Cronbach, confiabilidade composta e variância extraída indicaram bons valores de fidedignidade. Diferenças nos escores de autoestima em função do sexo e da idade não foram encontradas. Conclui-se que a EAR apresenta qualidades psicométricas satisfatórias, mostrando-se um instrumento confiável para medir autoestima em adolescentes brasileiros.
\end{abstract}

Palavras-chave: Autoestima, Autoavaliação, Psicometria.

\section{Rosenberg Self-Esteem Scale (RSS): factorial validity and internal consistency}

\begin{abstract}
The aim of this study was to investigate the psychometrics properties of the Rosenberg Self-Esteem Scale (RSS) for adolescents. The sample was composed of 4.757 adolescents, with ages between 14 and 18 years old $(M=15.77 ; S D=1.22)$ in nine Brazilian cities. Participants responded to an adapted version of the RSS for Brazil. Exploratory factorial analysis showed a bidimensional structure, with $51.4 \%$ of explained variance. This result was supported by confirmatory factor analysis. The internal consistency analysis by Cronbach alpha coefficient, composite reliability and extracted variance indicated good reliability. Differences in self-esteem for gender and age were not found. These findings show that RSS has satisfactory psychometric qualities and it's a reliable instrument to assess self-esteem in Brazilian adolescents.

Keywords: Self-esteem, Self-evaluation, Psychometrics.
\end{abstract}

Entende-se por autoestima um conjunto de sentimentos e pensamentos do indivíduo sobre seu próprio valor, competência e adequação, que se reflete em uma atitude positiva ou negativa em relação a si mesmo (Rosenberg, 1965). Coopersmith (1989) ressalta que o ponto fundamental da autoestima é o aspecto valorativo, o que influencia na forma como o indivíduo elege suas metas, aceita a si mesmo, valoriza o outro e projeta suas expectativas para o futuro (Bednar \& Peterson, 1995).

A autoestima relaciona-se a construtos psicológicos como bem-estar (Sánchez \& Barrón, 2003) e autoconceito (Martín-Albo, Núñez, Navarro \& Grijalvo, 2007), sendo amplamente investigada no campo da psicologia da personalidade (Schmitt \& Allik, 2005). Alguns pesquisadores têm visto a autoestima como traço (refletindo estabilidade durante um período de tempo) e estado (refletindo uma resposta a situações ou eventos de vida) (Harter \& Whitesell, 2003); enquanto outros sugerem que o desenvolvimento deste

\footnotetext{
1 Endereço para correspondência:

Universidade Federal do Rio Grande do Sul, Instituto de Psicologia Departamento de Psicologia do Desenvolvimento e da Personalidade

Rua Ramiro Barcelos, 2.600/ sala 115 - 90035-003 - Porto AlegreRS - Brasil.

E-mail: julianasbicigo@gmail.com
}

atributo possui descontinuidades ao invés de um curso estável ao longo do ciclo vital (Cole e cols., 2001).

A autoestima é considerada um dos principais preditores de resultados favoráveis na adolescência e na vida adulta, tendo implicações em áreas como sucesso ocupacional, relacionamentos interpessoais e desempenho acadêmico (Trzesniewski, Donnellan \& Robins, 2003). Por outro lado, a influência desta característica também tem sido observada em problemas adversos como agressão, comportamento antissocial e delinquência na juventude (Donnellan, Trzesniewski, Robins, Moffitt \& Caspi, 2005).

Dada a sua relação com o ajustamento psicossocial, a autoestima tem sido vista como um importante indicador de saúde mental e um fator relevante nas análises de crescimento e progresso nos países desenvolvidos (Mruk, 1995). Adicionalmente, a avaliação desta característica vem sendo considerada uma ferramenta importante na identificação e na prevenção de problemas psicológicos (Fan \& Fu, 2001).

A mensuração da autoestima tem sido mundialmente realizada por meio da Escala de Autoestima de Rosenberg - EAR (Rosenberg, 1965), conceitualizada pelo autor como um instrumento unidimensional capaz de classificar o nível de autoestima em baixo, médio e alto. A baixa autoestima se expressa pelo sentimento de incompetência, 
inadequação e incapacidade de enfrentar os desafios; a média é caracterizada pela oscilação do indivíduo entre o sentimento de aprovação e rejeição de si; e a alta consiste no autojulgamento de valor, confiança e competência (Rosenberg, 1965). A escala original foi desenvolvida para adolescentes e possui dez sentenças fechadas, sendo cinco referentes à "autoimagem" ou "autovalor" positivos e cinco referentes à "autoimagem negativa" ou "autodepreciação". As sentenças são dispostas no formato Likert de quatro pontos, variando entre "concordo totalmente" e "discordo totalmente".

A EAR foi traduzida para 28 idiomas e sua difusão em até 53 países tem revelado problemas referentes à estrutura unidimensional (Schmitt \& Allik, 2005). Vários pesquisadores realizaram a análise fatorial dos itens da escala e encontraram um construto bidimensional, que se reflete nas imagens positiva e negativa que o indivíduo possui de si mesmo (Avanci, Assis, Santos \& Oliveira, 2007; Hatcher \& Hall, 2009; Rojas-Barahona, Zegers \& Förster, 2009). Contudo, também há evidências confirmando o modelo de um fator para a EAR, o que torna sua estrutura fatorial uma questão controversa (Aluja, Rolland, García \& Rossier, 2007; Martín-Albo e cols., 2007; Schmitt \& Allik, 2005; Sinclair e cols., 2010).

Do ponto de vista teórico, a dimensionalidade da autoestima também vem sendo questionada. Autores como Harter (1999) e Coopersmith (1989), que há décadas têm estudado este conceito, sustentam o seu caráter multidimensional. Isso porque, além de uma possível decomposição do construto em fatores, os autores consideram importante avaliar esta característica em diferentes domínios (social, escolar, familiar, etc.), já que os sujeitos podem ter percepções positivas de si em algumas áreas e não em outras.

Ainda que existam controvérsias sobre $\mathrm{O}$ conceito de autoestima e sua estrutura fatorial, a EAR tem apresentado bons índices de consistência interna em pesquisas internacionais, que foram realizadas com amostras de adolescentes e adultos (Aluja e cols., 2007; Donnellan e cols., 2005). A adolescência tem sido a faixa etária mais pesquisada, sendo esta marcada por intensas mudanças físicas, sociais e cognitivas, que estão ligadas à construção e manutenção dessa característica psicológica (Robins, Hendin \& Trzesniewski, 2001).

Harter (1999) destaca que, na adolescência, a noção do autovalor torna-se um aspecto central porque os indivíduos desenvolvem capacidades cognitivas que lhes permitem realizar abstrações a respeito do self. Nesse período, o jovem também passa a atribuir maior importância à percepção que os outros têm sobre ele, $\mathrm{o}$ que parece levar, segundo pesquisas, a uma redução nos níveis de autoestima na adolescência inicial e média
(Clay, Vignoles \& Dittmar, 2005; Heaven \& Ciarrochi, 2008). Contudo, os achados são inconclusivos, pois um estudo longitudinal, por exemplo, indicou que jovens americanos tiveram um aumento de autoestima na adolescência inicial, seguido de decréscimo na adolescência média, havendo novamente um aumento no final da adolescência (Baldwin \& Hoffmann, 2002).

Quanto ao gênero, Harter (1999) explica que as meninas apresentam mais conflitos durante $\mathrm{O}$ desenvolvimento da autoimagem, pois valorizam mais os relacionamentos interpessoais e a opinião das outras pessoas a seu respeito, o que pode levá-las a índices mais baixos de autoestima. No entanto, não há consenso nas pesquisas quanto à existência de diferença neste atributo em adolescentes do sexo masculino e feminino. Pesquisas realizadas com adolescentes na Austrália e no Reino Unido indicaram um maior decréscimo de autoestima nas jovens (Heaven \& Ciarrochi, 2008; Robins \& Trzesniewski, 2005). Estudos realizados na Espanha e Europa mostraram que a noção de autovalor dos meninos foi apenas um pouco mais elevada que a das meninas (Martin-Albo e cols., 2007; Veleska e cols., 2009). Por outro lado, dois estudos de metanálise não revelaram diferenças de gênero para autoestima na adolescência (Gentile e cols., 2009; Twenge \& Campbell, 2001).

$\mathrm{Na}$ literatura nacional, entretanto, o construto autoestima tem sido pouco investigado, especialmente em estudos realizados com amostras com grande número de participantes, $\mathrm{o}$ que dificulta $\mathrm{O}$ conhecimento deste aspecto em nossa população (Avanci e cols., 2007; Bandeira, Arteche \& Reppold, 2008). Dentre as pesquisas nacionais encontradas, algumas apontam relações entre autoestima e gênero na adolescência, indicando escores ligeiramente superiores em jovens do sexo masculino (Romano, Negreiros \& Martins, 2007; Santos \& Maia, 2003). Com relação à estrutura fatorial da EAR, os estudos realizados no Brasil têm refletido as divergências encontradas na literatura, já que existem evidências corroborando tanto a unidimensionalidade da escala (Hutz, 2000; Santos \& Maia, 2003), quanto a existência de uma estrutura bifatorial (Andrade, Sousa \& Minayo, 2009; Avanci e cols., 2007; Romano e cols., 2007).

Tendo em vista essas considerações, o objetivo deste estudo foi avaliar as características psicométricas da EAR em 4.757 adolescentes brasileiros. Além disso, buscou-se verificar possíveis diferenças nos escores dos adolescentes em relação ao sexo e à idade.

\section{Método}

Participantes

Os dados deste estudo procedem do banco de dados da Pesquisa Nacional sobre Fatores de Risco e 
Proteção na Juventude Brasileira (Koller, CerqueiraSantos, Morais \& Ribeiro, 2005), que foi realizado com jovens brasileiros em situação de vulnerabilidade social. Participaram jovens das cidades de Arcos, Belo Horizonte, Brasília, Campo Grande, Maués, Porto Alegre, Presidente Prudente, Recife e São Paulo. Para composição da amostra, foi consultado o Índice de Desenvolvimento Humano (IDH), nas cidades onde este índice estava disponível, ou foram utilizados os critérios do Instituto Brasileiro de Geografia e Estatística (Censo) para determinar quais eram os bairros de nível socioeconômico baixo. A partir do levantamento dos bairros, foram sorteadas escolas públicas onde os dados foram coletados. Desse modo, foram participantes da pesquisa 4.757 adolescentes (54\% do sexo feminino), com idades entre 14 e 18 anos $(M=15,77 ; \quad D P=1,22)$, oriundos das regiões Norte $(3,7 \%)$, Nordeste $(14,2 \%)$, Noroeste $(15,5 \%)$, CentroOeste $(11,6 \%)$, Sudeste $(41,5 \%)$ e Sul $(13,5 \%)$ do Brasil. Cerca de $36 \%$ dos jovens estavam cursando entre a quarta e oitava série do ensino fundamental e $64 \%$ estavam cursando o ensino médio.

\section{Instrumento}

A Escala de Autoestima de Rosemberg (1965), utilizada neste estudo, baseia-se na adaptação de Hutz (2000) para o Brasil. Possui dez itens, sendo seis referentes a uma visão positiva de si mesmo e quatro referentes a uma visão autodepreciativa. As opções de resposta são "discordo", "nem concordo, nem discordo" e "concordo". A disposição dos itens no formato Likert de três pontos foi realizada para facilitar a compreensão dos adolescentes. Diversos pesquisadores têm utilizado versões modificadas da EAR, utilizando um menor ou maior número de itens ou itens reescritos, e opções de resposta variando entre o formato Likert de três e seis pontos, conforme os objetivos da pesquisa e a população estudada (Greenberger, Chuansheng, Dmitrieva \& Farruggia, 2003; Owens, 1994; Pesqueiro, 2005; Zimprich, Perren \& Hornung, 2005). A EAR apresentou boa consistência interna no estudo de validação original, realizado com 5.024 jovens de Nova Iorque $(\alpha=0,92)$, e em pesquisas brasileiras, nas quais o coeficiente alfa de Cronbach variou entre 0,68 (Avanci e cols., 2007) e 0,86 (Hutz, 2000; Santos \& Maia, 2003). Com relação à pontuação, quanto maior o escore obtido na escala, maior o nível de autoestima do indivíduo.

\section{Procedimentos}

Os jovens foram abordados em escolas públicas das nove cidades participantes. A aplicação da EAR foi realizada de forma coletiva, juntamente com outros instrumentos, por uma equipe de pesquisadores em cada cidade pesquisada, o que teve duração de, no máximo, 120 minutos. Os adolescentes e seus pais assinaram um Termo de Consentimento Livre e Esclarecido, e foi dada a garantia do sigilo das informações pessoais. O Estudo Nacional sobre Fatores de Risco e Proteção na Juventude Brasileira foi aprovado pelo Comitê de Ética da universidade das autoras.

\section{Análise de dados}

Com o objetivo de verificar a validade de construto da EAR, realizou-se uma análise fatorial exploratória (AFE), utilizando o método de extração dos componentes principais e rotação oblimin. Para verificar o número de fatores a serem retidos, foram utilizados os critérios de Kaiser (eigenvalue $>1$ ), Cattell (scree plot) e de Horn (análise paralela). A associação entre os fatores foi calculada pela correlação de Pearson. Para investigar com maior rigor a adequação do modelo aos dados empíricos, realizou-se análise fatorial confirmatória (AFC) de primeira e segunda ordem. Foi utilizado o método de estimação Maximum Likelihood, tendo em vista quatro indicadores de qualidade de ajuste (Goodness-of-fit): Tucker-Lewis Index (TLI), Comparative Fit Index (CFI) e Root Mean Square Error Approximation (RMSEA). O relato desses índices de ajustamento é recomendado pela literatura e, além disso, eles apresentam relativa independência do tamanho da amostra (Jackson, Gillaspy Jr. \& PurcStephenson, 2009). Valores a partir de 0,90 são considerados satisfatórios para TLI e CFI. Para RMSEA, valores abaixo de 0,10 são aceitáveis e valores inferiores a 0,05 são considerados excelentes ajustes (Hair, Black, Babin, Anderson \& Tatham, 2009).

Cabe destacar que o teste Qui-Quadrado $\left(\mathrm{X}^{2}\right)$ também é um teste utilizado para verificar a adequação de modelos teóricos. Entretanto, o $\mathrm{X}^{2}$ apresenta limitações quando se tratam de amostras robustas, já que é fortemente influenciado pelo tamanho da amostra. $\mathrm{O} \mathrm{X}^{2}$ é indicado quando o número de participantes varia entre 100 e 200 . Fora deste intervalo o teste tende a produzir resultados não-confiáveis, rejeitando qualquer modelo em amostras grandes ou aceitando um modelo de ajuste ruim em amostras pequenas. Nesses casos, pesquisadores têm utilizado os demais indicadores para sustentar a análise confirmatória (Hair \& cols., 2009). Na pesquisa de Finney, Pieper e Barron (2004), realizada com 2.111 jovens, os autores suprimiram as estimativas de $\mathrm{X}^{2} \mathrm{e}$ utilizaram índices como o RMSEA e o CFI. Este procedimento foi adotado no presente estudo.

A consistência interna da escala foi primeiramente avaliada por meio do coeficiente alfa de Cronbach, tendo como ponto de corte o valor de 0,70 
(Hair e cols., 2009). Com base na análise fatorial confirmatória, a fidedignidade também foi medida a partir do cálculo de confiabilidade composta e de variância extraída. Essas medidas são utilizadas em equações estruturais e são consideradas mais rigorosas que o coeficiente alfa de Cronbach. A confiabilidade composta aponta o grau em que os indicadores do construto descrevem o construto latente comum. A variância extraída é uma medida de consistência interna complementar, que mede a quantidade geral de variância dos indicadores explicada pela variável latente. Valores superiores a 0,70 são aceitos para a confiabilidade composta e para a variância extraída são indicados valores acima de 0,50 (Hair e cols., 2009).

Finalmente, foi calculada a estatística descritiva (média e desvio padrão) dos escores dos participantes na escala. Para identificar possíveis diferenças na autoestima global para sexo e idade empregou-se, respectivamente, o Teste $t$ de Student para amostras independentes e a ANOVA Oneway utilizando diferentes grupos etários.

\section{Resultados}

Inicialmente, verificou-se a fatoriabilidade da matriz de correlação entre os itens da escala através do índice Kaiser-Meyer-Olkin (KMO), obtendo-se o valor 0,80 para a amostra estudada. A análise fatorial exploratória, utilizando o método dos componentes principais e rotação oblimin, indicou dois fatores com eigenvalues maiores do que 1,00, que foram 2,86 (fator 1 ) e 2,28 (fator 2), explicando $51,40 \%$ da variância total. $\mathrm{O}$ fator 1 agrupa itens relacionados à autoestima positiva $(1,4,7,8,9,10)$ e o fator 2 , itens que se referem à autoestima negativa $(2,3,5,6)$, explicando, respectivamente, $28,62 \%$ e $22,78 \%$ da variância total. Foi adotado o ponto de corte de 0,30 para interpretação das cargas fatoriais dos itens. A Tabela 1 apresenta os resultados.

Tabela 1 - Cargas fatoriais, comunalidades e variância explicada para os escores da EAR

\begin{tabular}{llll}
\hline Itens & Fator 1 & Fator 2 & $\underline{\underline{h}}^{2}$ \\
\hline 01. Sinto que sou uma pessoa de valor como as outras pessoas & $0,57^{*}$ & 0,06 & 0,32 \\
04. Sou capaz de fazer tudo tão bem como as outras pessoas & $0,60^{*}$ & 0,07 & 0,36 \\
07. Eu acho que tenho muitas boas qualidades & $0,76^{*}$ & 0,03 & 0,58 \\
08. Eu tenho motivos para me orgulhar na vida & $0,66^{*}$ & 0,05 & 0,44 \\
09. De um modo geral, eu estou satisfeito(a) comigo mesmo(a) & $0,75^{*}$ & 0,06 & 0,56 \\
10. Eu tenho uma atitude positiva com relação a mim mesmo(a) & $0,73^{*}$ & 0,03 & 0,52 \\
02. Eu sinto vergonha de ser do jeito que sou & 0,06 & $0,75^{*}$ & 0,57 \\
03. Às vezes, eu penso que não presto para nada & 0,04 & $0,75^{*}$ & 0,56 \\
05. Levando tudo em conta, eu me sinto um fracasso & 0,08 & $0,81^{*}$ & 0,66 \\
06. Às vezes, eu me sinto inútil & 0,06 & $0,75^{*}$ & 0,56 \\
\hline Valores próprios & 2,86 & $2,28^{*}$ \\
\hline Variância (total=51,40\%) & $28,62 \%$ & $22,78^{\circ}$ \\
\hline
\end{tabular}

Análise fatorial com o método dos eixos principais e rotação oblimin

A solução de dois fatores foi suportada pelo gráfico scree plot (Figura 1) e pela análise paralela. Nesta última, os dois primeiros eigenvalues obtidos pela análise fatorial exploratória $(2,86 ; 2,28)$ foram superiores aos dois primeiros obtidos em 1.000 matrizes de correlações randômicas $(1,07 ; 1,04)$ com o mesmo número de itens e mesmo tamanho de amostra. Salienta-se que o critério de análise paralela tem sido considerado o procedimento mais confiável na verificação do número de fatores a serem retidos (Hayton, Allen \& Scarpello, 2004). No que diz respeito ao relacionamento entre os fatores da autoestima, o coeficiente de correlação de Pearson indicou uma relação significativa, porém próxima de zero $(r=0,08$, $p<0,01)$. 


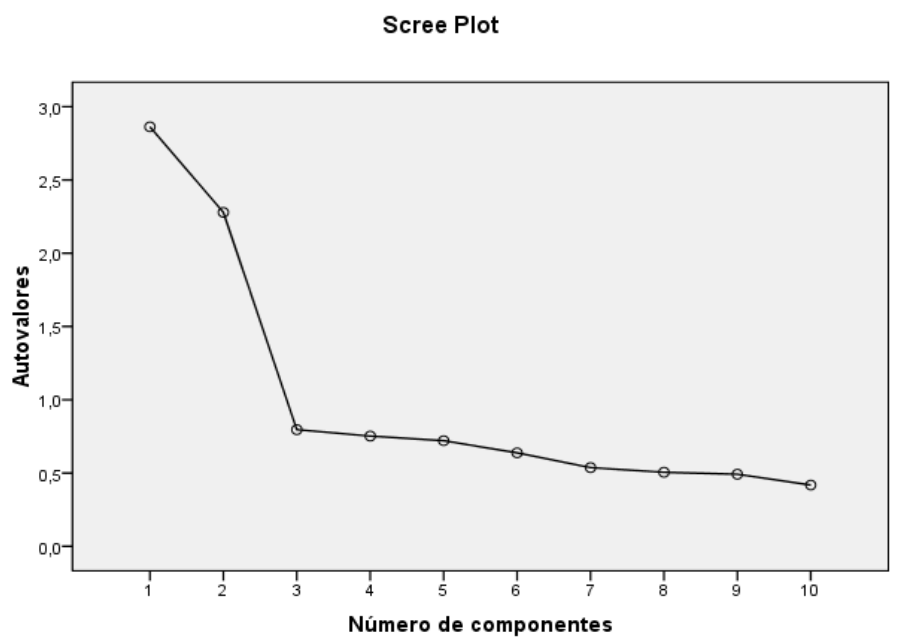

Figura 1 - Gráfico scree dos autovalores obtidos na análise fatorial exploratória da Escala de Autoestima de Rosenberg

A análise fatorial confirmatória corroborou o modelo bifatorial a partir de valores ideais para os índices de ajustamento utilizados (TLI=0,954; $\mathrm{CIF}=0,965$; RMSEA=0,048). Para reduzir as dúvidas quanto à estrutura fatorial encontrada, procedeu-se à análise do modelo unidimensional que, por sua vez, mostrou índices de adequação abaixo do ponto crítico (TLI=0,425; CIF=0,553; RMSEA=0,171). Com base nos resultados, testou-se um modelo hierárquico ou fatorial de segunda ordem, no qual os dois fatores (autoestima positiva e negativa) foram fixados como fatores de primeira ordem e uma variável latente (autoestima global) foi fixada como fator de segunda ordem. O modelo hierárquico apresentou índices virtualmente idênticos aos observados no modelo bifatorial (TLI=0,956; CFI=0,967; RMSEA=0,047).

De acordo com a literatura, um modelo hierárquico não seria capaz de produzir um modelo de ajuste melhor que um modelo de primeira ordem
(Arnau \& Thompson, 2000). Entretanto, um modelo de segunda ordem que rivaliza com o desempenho de um modelo de primeira ordem pode ser uma alternativa viável, dependendo do modelo teórico (Koufteros, Babbar \& Kaighobadi, 2009; Milfont e cols., 2008). Neste estudo, porém, manteve-se o modelo bifatorial, tendo em vista a fraca correlação entre os fatores da autoestima, que pode ter sido significativa em virtude do tamanho da amostra. Estudos corroborando uma estrutura unidimensional ou um modelo hierárquico com duas dimensões correlacionadas têm encontrado índices de correlação entre os fatores que variam de 0,20 a 0,70 (Roth, Decker, Herzberg \& Brähler, 2008; Schmitt \& Allik, 2005).

Quanto à análise de consistência interna, os valores obtidos utilizando o coeficiente alfa de Cronbach, confiabilidade composta e variância extraída podem ser observados na Tabela 2.

Tabela 2 - Indicadores de fidedignidade para a EAR

\begin{tabular}{lccc}
\hline Autoestima & Alfa de Cronbach & Confiabilidade composta & Variância extraída \\
\hline Positivo & 0,76 & 0,90 & 0,60 \\
Negativo & 0,77 & 0,82 & 0,53 \\
Global & 0,70 & & \\
\hline
\end{tabular}

$\mathrm{Na}$ análise descritiva da EAR, verificou-se que a média dos escores dos participantes foi de 23,33 $(D P=3,47)$. Os cálculos inferenciais utilizando teste $t$ de Student e a ANOVA Oneway não apontaram diferenças significativas na autoestima global para sexo e idade.

\section{Discussão}

Este estudo buscou verificar alguns parâmetros psicométricos da Escala de Autoestima de Rosenberg
(EAR) para adolescentes brasileiros. Quanto à validade de construto, a análise fatorial exploratória apontou uma estrutura bidimensional (positiva e negativa) por meio dos critérios convencionados (Kaiser, Cattell e Horn). A magnitude das cargas fatoriais e a variância explicada pelos fatores consistiram em bons indicadores de validade para a EAR. A análise fatorial confirmatória corroborou a superioridade do modelo bidimensional em relação ao unidimensional, com 
valores excelentes para os índices de ajustamento utilizados, revelando adequação dos dados empíricos ao modelo teórico. Um modelo hierárquico também poderia ser admitido, porém o modelo bifatorial foi considerado mais parcimonioso em razão da fraca correlação observada entre os fatores.

Com relação à confiabilidade, a EAR apresentou consistência interna satisfatória. Os resultados obtidos mediante o coeficiente alfa de Cronbach foram semelhantes aos observados em outros trabalhos como, por exemplo, aqueles realizados no Chile e no Brasil (Avanci e cols., 2007; Hutz, 2000; Rojas-Barahona e cols., 2009), sendo um pouco inferiores aos valores encontrados em outros países, os quais giraram em torno de 0,75 (Schmitt \& Allik, 2005). Nota-se, contudo, que o coeficiente alfa de Cronbach diminuiu quando os fatores foram considerados em conjunto, o que novamente sugere a bidimensionalidade da escala. Ainda quanto à fidedignidade, ressaltam-se os valores ideais obtidos no cálculo de confiabilidade composta, revelando que os indicadores do construto (autoestima positiva e autoestima negativa) contribuem substancialmente para a descrição do construto latente (autoestima global).

Em linhas gerais, as evidências referentes à dimensionalidade da EAR têm sido controversas e, para muitos pesquisadores, a identificação de dois fatores tem sido resultado dos efeitos do método (Greenberger e cols., 2003; Quilty, Oakman \& Risko, 2006). Marsh (1996) sustenta que as questões agrupadas em cada fator resultariam na tendência dos respondentes em concordar com as afirmações escritas de forma positiva e discordar daquelas que traduzem aspectos negativos do indivíduo. $O$ autor também enfatiza que os efeitos do método ocorrem principalmente em pessoas mais jovens, pois responder às afirmações negativas implica um maior nível de complexidade cognitiva do que responder aos itens positivos. Em um estudo com sete itens da EAR (quatro positivos e três negativos), Marsh (1996) demonstrou que estudantes com menos habilidade verbal foram especialmente suscetíveis a dar respostas a itens negativos que eram incoerentes com suas respostas aos itens positivos.

Os efeitos do método têm sido considerados não apenas pelos pesquisadores que apoiam a presença de dois fatores para a EAR, mas também por aqueles que defendem uma estrutura unifatorial. Assim, em algumas pesquisas, a escala é vista como unidimensional, mas são incluídos os efeitos do método para os itens negativos (Gana, Alaphilippe \& Bailly, 2005; Schmitt \& Allik, 2005), enquanto outros pesquisadores incluem esses efeitos para os itens positivos (Martín-Albo e cols., 2007; Wang, Siegal,
Falck \& Carlson, 2001). Também tem sido sustentado que o melhor ajustamento ao construto é obtido quando se consideram os efeitos do método tanto para os itens positivos quanto para os negativos (Quilty e cols., 2006; Tomás \& Oliver, 1999). Por outro lado, autores apoiam que a solução de dois fatores correlacionados possui melhor ajustamento ao construto (Greenberger e cols., 2003; Romano e cols., 2007).

O presente estudo reforça a perspectiva bidimensional para EAR (Avanci e cols., 2007; Greenberger e cols., 2003; Hatcher \& Hall, 2009; Rojas-Barahona e cols., 2009), porém fornece evidências acerca da independência entre as suas dimensões positiva e negativa. Contudo, a solução de dois fatores não-correlacionados pode ter sido resultado dos efeitos do método sobre os itens da escala. De acordo com pesquisadores (Marsh, 1996; Wright \& Stone, 2004) as respostas às sentenças negativas, mesmo após a sua inversão, produzem fatores independentes ao invés de dois pólos de uma mesma dimensão. Desse modo, pode ser recomendável analisar o escore total da EAR e também escores parciais para cada fator.

Por outro lado, é importante considerar que existem várias evidências sustentando a estrutura unifatorial para a referida escala (Aluja e cols., 2007; Sinclair e cols., 2010). Autores salientam que a inconsistência entre os achados ocorre porque o instrumento pode ser unidimensional em certas populações e bidimensional em outras (Marsh, 1996; Schmitt \& Allik, 2005). Schmitt e Allik (2005) verificaram que algumas culturas apresentaram maior viés para os itens negativos da EAR do que outras. Para os autores esse viés seria um artefato responsável pela aparência de dois fatores para o construto autoestima em alguns contextos.

Nessa perspectiva, Greenberger e cols. (2003) argumentam que os itens da escala deveriam ser reescritos na mesma direção, avaliando ou a "autoestima positiva" ou a "autoestima negativa". Em sua pesquisa, os autores compararam três versões da EAR (a versão original, uma versão com todos os itens escritos na forma positiva e uma versão com todos os itens escritos na forma negativa) e avaliaram a validade de construto com instrumentos que avaliam depressão e negação (self-deception). A escala original foi a única a apresentar estrutura bifatorial e, além disso, apresentou maior sobreposição aos construtos de depressão e negação do que as outras duas versões. Para Greenberger e cols. (2003), é necessário reformular a EAR a fim de que o instrumento adquira uma estrutura unidimensional, conforme foi proposto por Rosenberg. 
Relações entre autoestima, idade e gênero não foram encontradas neste estudo, o que também aconteceu na pesquisa brasileira de Weber, Stasiack e Brandenburg (2003) e em estudos internacionais (Gentile e cols., 2009; Twenge \& Campbell, 2001). Este achado não corrobora a hipótese de que as meninas tendem a apresentar menores níveis de autoestima e sugere que este atributo não está necessariamente associado ao gênero e à idade na adolescência. Contudo, são necessárias novas pesquisas para clarificar essa questão, tendo em vista que relações entre essas variáveis foram identificadas em outros estudos brasileiros (Romano e cols., 2007; Santos \& Maia, 2003).

\section{Considerações finais}

Esta pesquisa identificou boas propriedades psicométricas para a EAR no Brasil. Verificou-se uma estrutura bifatorial com fatores independentes para escala, sugerindo-se o cálculo de escores separados para cada fator da autoestima. Entretanto, os resultados deste estudo devem ser interpretados com cautela. Em primeiro lugar, utilizou-se uma versão adaptada da EAR, com três itens na escala Likert ao invés de quatro, sendo possível que resultados diferentes sejam obtidos utilizando-se a versão original. Embora seja necessário adaptar medidas de autoestima para cada contexto, a utilização de versões modificadas dificulta a comparação entre os resultados. Em segundo lugar, o estudo foi realizado com uma amostra específica, ou seja, com adolescentes de 14 a 18 anos, de nível socioeconômico baixo. Desse modo, embora a amostra utilizada tenha sido composta por um grande número de adolescentes, representando todas as regiões do país, os achados não podem ser generalizados para toda a população de adolescentes brasileiros. Finalmente, as características da amostra pesquisada podem ter acentuado os efeitos do método sobre os itens da EAR.

Ainda que existam questões controversas acerca dos efeitos do método sobre a EAR, acredita-se que isso apenas estimula a realização de novas pesquisas. Estudos verificando a validade de construto da escala com outros instrumentos de aferição psicológica, correlacionando estes últimos com os fatores positivo e negativo da autoestima contribuirão para esta discussão. Sugere-se ainda que sejam realizados estudos longitudinais para verificar a estabilidade da autoestima durante a adolescência. Investigações com amostras clínicas também são recomendadas, já que pouco se sabe sobre a eficácia da EAR no âmbito diagnóstico.

\section{Referências}

Aluja, A., Rolland, J., García, L. \& Rossier, J. (2007). Dimensionality of the Rosenberg Self-Esteem Scale and its relationships with the three and the five-factor personality models. Journal of Personality Assessment, 88(2), 1-4.

Andrade, E., Sousa, E. \& Minayo, M. C. (2009). Selfesteem and quality of life: essential for the mental health of police officers. Ciência e Saúde Coletiva, 14(1), 275-285.

Arnau, R. C. \& Thompson, B. (2000). Second-order confirmatory factor analysis of the WAIS-III. Assessment, 7(3), 237-246.

Avanci, S., Assis, S., Santos, N. \& Oliveira, R. (2007). Adaptação transcultural da Escala de Autoestima de Rosenberg para adolescentes. Psicologia: Reflexão e Crítica, 20(3), 397-405.

Baldwin, S. A. \& Hoffmann, J. P. (2002). The dynamics of self-esteem: a growth-curve analysis. Journal of Youth and Adolescence, 31(2), 101-113.

Bandeira, D., Arteche, A. \& Reppold, C. (2008). Escala de Autopercepção de Harter para adolescentes: um estudo de validação. Psicologia: Teoria e Pesquisa, 24(3), 341-345.

Bednar, R. \& Peterson, S. (1995). Self-esteem: paradoxes and innovation in clinical theory and practice. Washington, D.C.: American Psychological Association.

Clay, D., Vignoles, V. \& Dittmar, H. (2005). Body image and self-esteem among adolescent girls: testing the influence of sociocultural factors. Journal of Research on Adolescence, 15, 451-477.

Cole, D. A., Maxwell, S. E., Martin, J. M., Peeke, L. G., Seroczynski, A. D., Tram, J. M., Hoffman, K. B., Ruiz, M. D., Jacquez, F. \& Maschman, T. (2001). The development of multiple domains of child and adolescent self-concept: a cohort sequential longitudinal design. Child Development, 72, 17231746.

Coopersmith, S. (1989). Coopersmith Self-esteem Inventory. Palo Alto: Consulting Psychologists Press.

Donnellan, M., Trzesniewski, K., Robins, R., Moffitt, T. \& Caspi, A. (2005). Low self-esteem is related to aggression, antisocial behavior, and delinquency. Psychological Science, 16(4), 328-335.

Fan, F. \& Fu, J. (2001). Self-concept and mental health of college students. Chinese Mental Health Journal, 15, 76-77. 
Finney, S. J., Pieper, S. L. \& Barron, K. E. (2004). Examining the psychometric properties of the Achievement Goal Questionnaire in a general academic context. Educational and Psychological Measurement, 64, 365-382.

Gana, K., Alaphilippe, D. \& Bailly, N. (2005). Factorial structure of the french version of the Rosenberg Self-Esteem Scale among the elderly. International Journal of Testing, 5(2), 169-176.

Gentile, B., Grabe, S., Dolan-Pascoe, B., Twenge, J. M., Wells, B. E. \& Maitino, A. (2009). Gender differences in domain-specific self-esteem: a metaanalysis. Review of General Psychology, 13, 34-45.

Greenberger, E., Chuansheng, C., Dmitrieva, J. \& Farruggia, S. (2003). Item-wording and the dimensionality of the Rosenberg Self-Esteem Scale: do they matter? Personality and Individual Differences, 35, 1241-1254.

Hair, J. F., Black, W. C., Babin, B. J., Anderson, R. E. \& Tatham, R. L. (2009). Análise multivariada dos dados. $6^{a}$ ed. Porto Alegre: Bookman.

Harter, S. (1999). The construction of the self: a developmental perspective. Nova Iorque: Guilford Press.

Harter, S. \& Whitesell, N. R. (2003). Beyond the debate: why some adolescents report stable selfworth over time and situation, whereas others report changes in self-worth. Journal Personality, 71, 1027-1058.

Hatcher, J. \& Hall, L. (2009). Psychometric properties of the Rosenberg Self-Esteem Scale in African American single mothers. Issues in Mental Health Nursing, 30(2), 70-77.

Hayton, J. C., Allen, D. G. \& Scarpello, V. (2004). Factor retention decisions in exploratory factor analysis: a tutorial on parallel analysis. Organizational Research Methods, 7, 191-205.

Heaven, P. \& Ciarrochi, J. (2008). Parental styles, gender and the development of hope and selfesteem. European Journal of Personality, 22, 707-724.

Hutz, C. (2000). Adaptação brasileira da Escala de Autoestima de Rosenberg. Curso de Pós-Graduação em Psicologia, Universidade Federal do Rio Grande do Sul, Porto Alegre, Mimeo.

Instituto Brasileiro de Geografia e Estatística - IBGE (2000). Censo demográfico 2000. Obtido em 12 de junho de 2006 do World Wide Web: $<$ http://www.ibge.gov.br/censo $>$.

Jackson, D. L., Gillaspy Jr., J. A. \& Purc-Stephenson, R. (2009). Reporting pratices in confimatory factor analysis: an overview and some recommendations. Psychological Methods, 14, 6-23.

Koller, S. H., Cerqueira-Santos, E., Morais, N. A. \& Ribeiro, J. (2005). Juventude brasileira. Relatório técnico. Washington DC: World Bank.

Koufteros, X., Babbar, S. \& Kaighobadi, M. (2009). A paradigm for examining second-order factor models employing structural equation modeling. International Journal of Production Economics, 120, 633652.

Marsh, H. (1996). Positive and negative global selfesteem: a substantively meaningful distinction or artifactors? Journal of Personality and Social Psychology, 70, 818-819.

Martín-Albo, J., Núñez, J., Navarro, J. \& Grijalvo, F. (2007). The Rosenberg Self-Esteem Scale: translation and validation in university students. The Spanish Journal of Psychology, 10(2), 458-467.

Milfont, T., Gouveia, V., Jesus, G. R., Gusmão, E. E. da S., Chaves, S. S. da S. \& Coelho, J. A. P. de M. (2008). Estrutura fatorial da Escala de Atitudes frente a relacionamentos afetivos estáveis. Psicologia: Teoria e Pesquisa, 24(3), 331-339.

Mruk, C. (1995). Self-esteem: research, theory, and practice. New York: Springer.

Owens, T. (1994). Two dimensions of self-esteem: reciprocal effects of positive self-worth and selfdeprecation on adolescent problems. American Sociological Review, 59, 391-407.

Pesqueiro, A. (2005). Uso de prótese dentária total por idosos: aspectos psicológicos. (Dissertação de Mestrado). Goiás: Universidade Católica de Goiás - PróReitoria de Pós-Graduação e Pesquisa.

Quilty, L., Oakman, J. \& Risko, E. (2006). Correlates of the Rosenberg Self-Esteem Scale method effects. Structural Equation Modeling, 13, 99-117.

Robins, R. \& Trzesniewski, K. (2005). Self-esteem development across the life span. Current Directions in Psychological Science, 14(3), 158-162.

Robins, R., Hendin, H. \& Trzesniewski, K. (2001). Measuring global self-esteem: construct validation of a single-item measure and the Rosenberg SelfEsteem Scale. Personality and Social Psychology Bulletin, 27, 151-161.

Rojas-Barahona, C., Zegers, B. \& Förster, C. (2009). La Escala de Autoestima de Rosenberg: validación para Chile en una muestra de jóvenes adultos, adultos y adultos mayores. Revista Médica do Chile, 137, 791-800. 
Romano, A., Negreiros, J. \& Martins, T. (2007). Contributos para validação da Escala de Autoestima de Rosenberg numa amostra de adolescentes da região interior norte do país. Psicologia, Saúde \& Doenças, 8(1), 109-116.

Rosenberg, M. (1965). Society and the adolescent self-image. Princeton: Princeton University Press.

Roth, M., Decker, O., Herzberg, P. \& Brähler, E. (2008). Dimensionality and norms of the Rosenberg Self-esteem Scale in a German general population sample. European Journal of Psychological Assessment, 24(3), 190-197.

Sánchez, E. \& Barrón, A. (2003). Social psychology of mental health: the social structure and personality perspective. The Spanish Journal of Psychology, 6, 311.

Santos, P. \& Maia, J. (2003). Análise factorial confirmatória e validação preliminar de uma versão portuguesa da Escala de Auto-Estima de Rosenberg. Psicologia: Teoria, Investigação e Prática, 2, 253-268.

Schmitt, D. \& Allik, J. (2005). Simultaneous administration of the Rosenberg Self-Esteem Scale in 53 nations: exploring the universal and culture-specific features of global self-esteem. Journal of Personality and Social Psychology, 89, 623642.

Sinclair, S., Blais, M., Gansler, D., Sandberg, E., Bistis, K. \& LoCicero, A. (2010). Psychometric properties of the Rosenberg Self-Esteem Scale: overall and across demographic groups living within the United States. Evaluation \& the Health Professions, 33(1), 56-80.
Tomás, J. \& Oliver, A. (1999). Rosenberg's SelfEsteem Scale: two factors or method effects. Structural Equation Modeling, 6, 84-98.

Trzesniewski, K., Donnellan, M. \& Robins, R. (2003). Stability of self-esteem across the life span. Journal of Personality and Social Psychology, 84, 205-220.

Twenge, J. \& Campbell, W. (2001). Age and birth cohort differences in self-esteem: a cross-temporal meta-analysis. Personality and Social Psychology Review, 5, 321-344.

Veleska, Z., Geckova, A., Gajdosova, B., Orosova, O., VanDijk, J. \& Reijneveld, S. (2009). Self-esteem and resilience: the connection with risky behavior among adolescents. Addictive Behaviors, 34, 287291.

Wang, J., Siegal, H., Falck, R. \& Carlson, R. (2001). Factorial structure of Rosenberg's Self-Esteem Scale among crack-cocaine drug users. Structural Equation Modeling, 8, 275-286.

Weber, L., Stasiack, G. \& Brandenburg, O. (2003). Percepção da interação familiar e auto-estima de adolescentes. Aletheia, 17/18, 95-105.

Wright, B. \& Stone, M. (2004). Making measures. Chicago: The Phaneron Press.

Zimprich, D., Perren, S. \& Hornung, R. (2005). A twolevel confirmatory factor analysis of a Modified Rosenberg Self-Esteem Scale. Educational and Psychological Measuremen, 65, 465-481.

Recebido em junbo de 2010 Reformulado em setembro de 2010 Aprovado em novembro de 2010

Sobre as autoras:

Juliana Burges Sbicigo é psicóloga, graduada em Psicologia pela Universidade do Vale do Rio dos Sinos (UNISINOS). Está cursando Mestrado em Psicologia, com bolsa CNPq, pelo Programa de Pós-graduação da UFRGS. É membro do Núcleo de Estudos e Pesquisas em Adolescência (NEPA/UFRGS). Possui interesse pelo estudo na área de Psicologia do Desenvolvimento Humano e Avaliação Psicológica (Psicometria).

Denise Ruschel Bandeira é psicóloga e especialista em Diagnóstico Psicológico (PUC-RS). Mestrado e Doutorado em Psicologia (UFRGS). Atualmente é professora associada da UFRGS. Tem experiência na área de Construção e Validação de Testes. É coordenadora pedagógica do Curso de Especialização em Psicologia Clínica com Ênfase em Avaliação Psicológica (UFRGS) e editora-geral da Revista Psicologia: Reflexão e Crítica.

Débora Dalbosco Dell'Aglio é psicóloga, mestre e doutora em Psicologia do Desenvolvimento (UFRGS), docente do Programa de Pós-Graduação em Psicologia da Universidade Federal do Rio Grande do Sul, coordenadora do Núcleo de Estudos e Pesquisas em Adolescência (NEPA/UFRGS) e pesquisadora do CNPq com as seguintes áreas de interesse: Desenvolvimento Humano, Famílias, Adolescentes e Crianças em Situação de Risco e de Institucionalização. 
